\title{
ULTRACAM EAGLE, DETAILS AND INSIGHT
}

\author{
Michael Gruber, Martin Ponticelli, Richard Ladstädter \\ Alexander Wiechert \\ Vexcel Imaging Austria / Microsoft Photogrammetry \\ Anzengrubergasse 8/4, 8010 Graz / Austria \\ \{michgrub, mponti, rladstae, alwieche\}@microsoft.com
}

\section{Commission I/2， LIDAR, SAR and Optical Sensors for Airborne and Spaceborne Platforms}

\author{
KEY WORDS: Photogrammetry, Digital, Camera, Large Format, Mapping
}

\begin{abstract}
UltraCam Eagle, the ultra large format digital aerial frame camera is the latest product out of a family of innovative mapping cameras by Microsoft/Vexcel. The sensor was introduced in 2011 and was well accepted by the community. The ultra large frame format of 20010 pixels cross track and 13080 pixels along the flight line offers a high productivity in the air. The sensor head consists of new CCD sensor arrays, new optical lens systems and new electronic components. In this contribution we present the camera design, technical parameters and the result from different flight missions.
\end{abstract}

\section{Introduction}

The first product of this camera family was introduced into the market in May 2003 under the name UltraCam D. This digital frame camera did show a very unique design and offered an image format of almost 90 Mega Pixels. Since about 10 years Microsoft/Vexcel was able to continuously develop and enhance this camera product, the software to operate the camera and to process images as well as the photogrammetric production software. In May 2011 the new flagship product, the UltraCam Eagle - a 260 Megapixel digital aerial framing camera was presented. New camera electronic components, new lens systems and a high performance CCD sensor were designed for this camera. After a year of operation we present our experience and results from photo missions and focus on the specific features of this camera. Productivity in the air, high frame rate and unmatched image quality are highlights of this product. The basic concept of the camera system - the multi cone design - did not change. Even in a time when large CCD sensors beyond the 100 Mega Pixel frame size are available this concept has strong advantages which are consequently exploited. The camera is equipped with 8 individual cones - four are responsible for the large format panchromatic image. This 20010 Pixel by 13080 Pixel digital frame image is processed from 9 CCD sensor arrays. Four additional camera heads contribute to the multispectral image adding red, green, blue and near infrared information. This concept leads to a highly parallel system architecture and therefore to a fast image cycle. Thus a high image overlap at large scale is made available by this camera. In this contribution we present a short overview on the software which is part of the camera system. The so-called stitching concept is basically responsible for the post processing of the raw image data This data set is acquired during the photo mission. The stitching software exploits concepts of image analysis and least squares adjustment in order to transform the raw image data set into one single frame. This software was continuously improved and conceptual details as well as the results were reported. During this processing step the camera calibration data set introduces all necessary camera parameters. The calibration process is a highly redundant procedure were some ten-thousand image measurements contribute to the geometric and radiometric description of the camera.

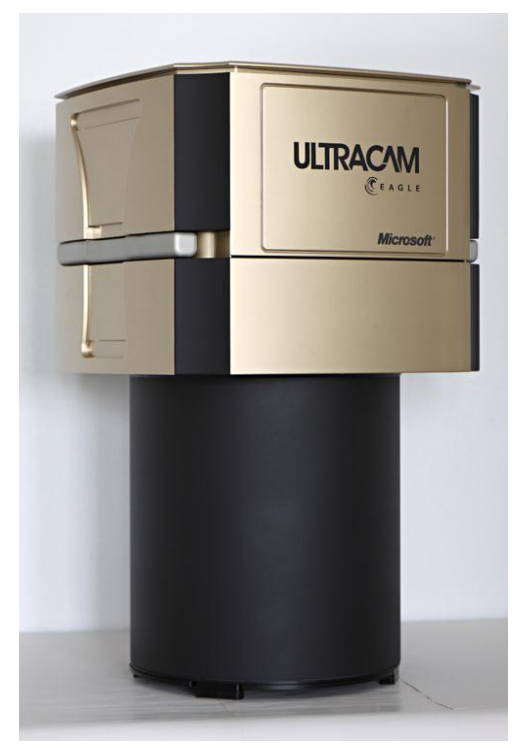

Figure 1: UltraCam Eagle digital aerial frame camera. 
The ultra large frame format of the UltraCam Eagle offers an increase of efficiency of $+70 \%$ compared to the UltraCam D from 2003 (cf. Figure 2).
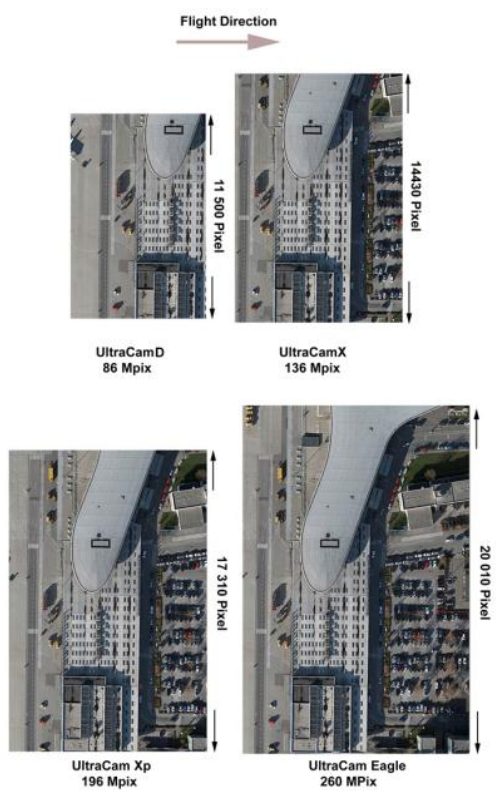

Figure 2: UltraCam frame format: From upper left to lower right UltraCam D (11500 Pixel cross track), UltraCam X, UltraCam Xp and UltraCam Eagle (20010 Pixels cross track)

\section{The UltraCam Sensor Design}

The design of the UltraCam Sensor head is based on a multi cone concept. Four cones acquire the large panchromatic frame and four additional cones acquire the red, green, blue and near infrared bands (cf. Figure 3a). The basic idea was introduced in (Leberl et al., 2003) and consists of the four cone concept for the large format panchromatic image (cf. Figure $3 b$ ).

This camera design is successfully supported by software to manage the sensor (the Camera Operating Software) and to process image data after the flight mission (embedded into the UltraMap software product). Major improvements of this software were reported, e.g. the so called "Monolitic Stitching" which was introduced in 2010 (Ladstädter et al., 2010).

The monolithic stitching software and the multi cone hardware design of the UltraCam sensors complement one another at a very effective manner. This was the guiding idea of the Vexcel product design - known as "Software Leveraged Hardware". Beyond the post processing of the acquired image data the UltraMap software offers the complete photogrammetric processing chain. This consists of the Aero-triangulation and Bundle Adjustment Module, the Color Adjustment Module and the new Surface and Ortho Module.

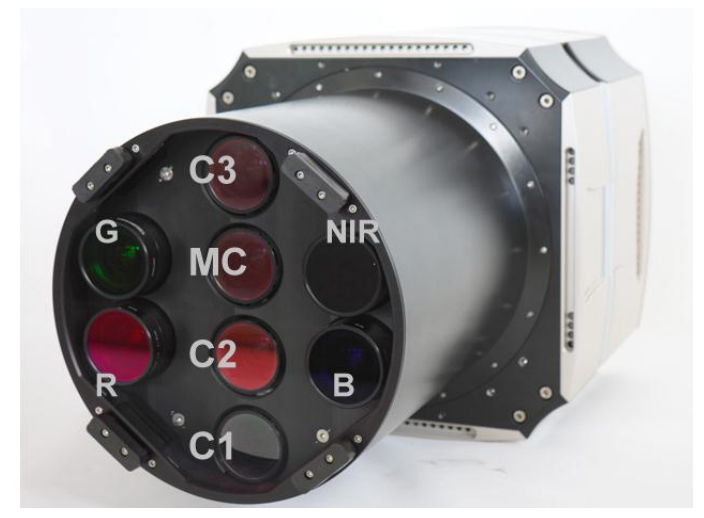

Figure 3a: UltraCam Eagle front plate with 4 cones for the panchromatic image (MC, C1,C2 and C3) and 4 cones for multispectral data acquisition $\mathrm{R}, \mathrm{G}, \mathrm{B}, \mathrm{NIR})$.

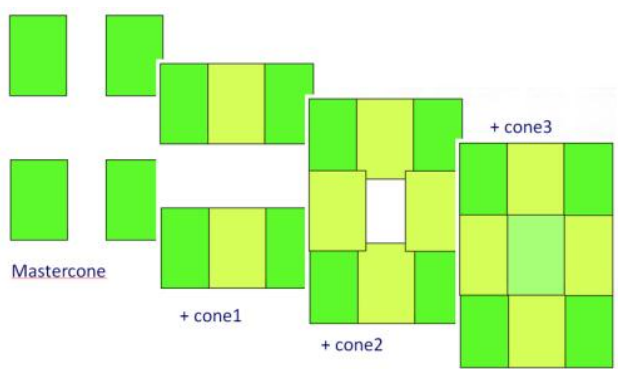

Figure 3b: UltraCam design concept. Four cones of the panchromatic partition are equipped with 9 CCD sensor arrays.

\section{UltraCam Eagle - Sensor Details}

New features and components make UltraCam Eagle a completely new sensor and separate it from its predecessors. Almost all hardware components were redesigned or newly developed and contribute to the performance and the flexibility of the camera. The most important newly developed components of the UltraCam Eagle shall be listed in this order:

- The new proprietary electronic components have been developed at Vexcel Imaging to optimize the CCD sensor management and to enable a fast readout. Thus a frame rate of $1 / 1,8$ seconds can be achieved still keeping advantageous single-tap readout at increased CCD pixel count.

- $\quad$ The OEM CCD detector arrays at $5.2 \mu \mathrm{m}$ pixel pitch and $>12$ bit dynamic range allow to build the ultra large 260 Megapixel frame format with more than 20,000 pixel across the flight strip.

- The OEM optical system with $80 \mathrm{~mm}$ focal distance for PAN and $27 \mathrm{~mm}$ focal distance for RGB/NIR resolving the fine structure of the CCD detector arrays at $100 \mathrm{lp} / \mathrm{mm}$. 
- $\quad$ A second OEM optical system with $210 \mathrm{~mm}$ focal distance for PAN and $70 \mathrm{~mm}$ focal distance for $\mathrm{RGB} / \mathrm{NIR}$ at a resolving power of $100 \mathrm{lp} / \mathrm{mm}$ is tuned to go for high resolution mapping from high altitude flight missions.

- The ability to make use of exchangeable lens cones supported by the new precision lens mount and tools to enable the users to exchange lenses on site

- The integration of all on board IT components including SSD storage and computer boards as well as the integrated navigation solution UltraNav.

Key parameters of the UltraCam Eagle are listed in Table 1.

\begin{tabular}{lr}
\hline Panchromatic image size: & $20,010 \times 13,080$ pixels \\
Panchromatic physical pixel size: & $5.2 \mu \mathrm{m}$ \\
Input data quantity per image: & 842 MegaByte \\
& 260 MegaPixe
\end{tabular}

Lens system 1: $\quad 80 \mathrm{~mm}$ PAN and $27 \mathrm{~mm}$ RGBNIR

Lens system 2: $\quad 210 \mathrm{~mm}$ PAN and $70 \mathrm{~mm}$ RGBNIR exchangeable by a trained end user no recalibration required

Maximum frame rate:

$<1.8$ seconds per frame

CCD signal to noise ratio:

$72 \mathrm{~dB}$

CCD image dynamic:

14 bit

workflow dynamic:

16 bit

Physical dimensions with $80 \mathrm{~mm}(210 \mathrm{~mm})$ PAN lenses, including computer and storage module:

$43 \mathrm{~cm} \mathrm{x} 43 \mathrm{~cm} \mathrm{x} 76 \mathrm{~cm}(86 \mathrm{~cm})$

Weight with $80 \mathrm{~mm}(210 \mathrm{~mm})$ PAN lenses, including computer and storage module: approx. $75 \mathrm{~kg}(80 \mathrm{~kg})$

Power consumption at full performance, including computer and storage module: 350 watts

Solid-state disc pack with built-in data redundancy for maximum data reliability.

Unlimited with use of multiple data units with approximately 3.3 terabytes (3,800 images) per unit

Data recording time @ $10 \mathrm{~cm}$ GSD, 60 percent forward overlap,140 kts@8 hours per data unit

Maximum forward overlap @ 10 cm GSD (@ 5 cm GSD) with140 kts@90 percent (80 percent)

Table 1: UltraCam Eagle Features and Technical Details

\section{Geometric Performance}

The geometric performance of the UltraCam Eagle was analyzed by means of a least squares bundle adjustment of a block of images. We report about a flight mission which was carried out in our validation area $20 \mathrm{~km}$ east of the city of Graz, Austria on $22^{\text {nd }}$ February 2012. The block consists of 205 images, 5 flight lines North-South and 2 flight lines East-West. The flying altitude was about $2100 \mathrm{~m}$ ASL, thus an image scale of $1 / 21000$ at $80 \mathrm{~mm}$ FD and a GSD of about $10 \mathrm{~cm}$ was achieved. The overlap was set to $80 \%$ / $60 \%$. Thus the redundancy of the adjustment was high and the result of the data can be well accepted to reflect the quality of the camera (cf. Figure 4). Of interest was the analysis of remaining image residuals which could be identified at a magnitude of $0.8 \mu \mathrm{m}$ for both $\mathrm{x}$ and $\mathrm{y}$. (cf. Figure 5).

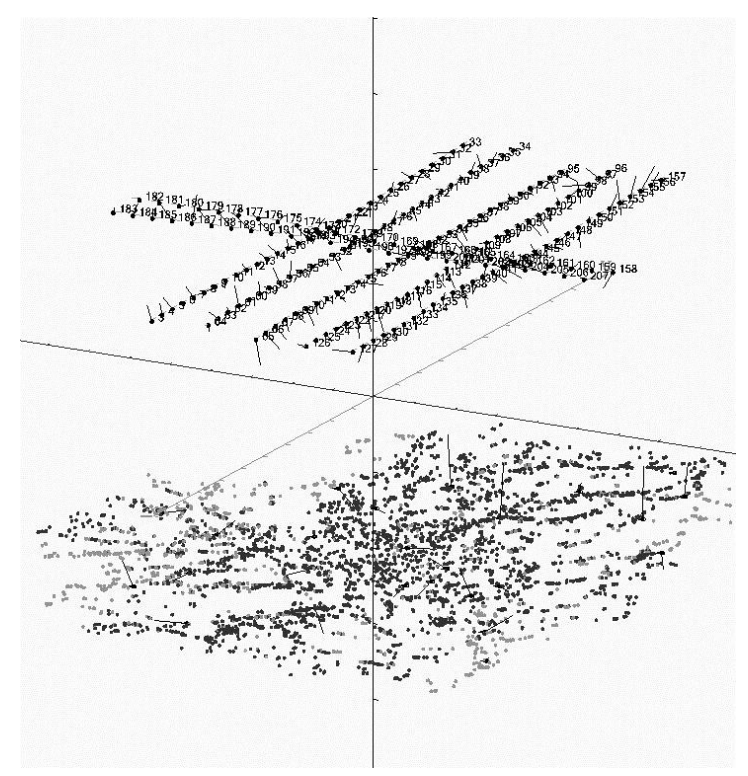

Figure 4: Aero-triangulation project from 205 UltraCam Eagle photos. The block layout shows the high overlap and cross strips ( $3 \mathrm{~d}$ view by BINGO). Sigma_o is at 0.86 .

The accuracy of the photogrammetric set up was verified by checkpoints and a RMS Error in $\mathrm{X}, \mathrm{Y}$ and $\mathrm{Z}$ at $28 \mathrm{~mm}$, $24 \mathrm{~mm}$ and $26 \mathrm{~mm}$ was identified. This corresponds to $1 / 3$ to $1 / 4$ of GSD for horizontal and vertical. The RMS Control Point Residuals are comparable and show a magnitude of $35 \mathrm{~mm}, 34 \mathrm{~mm}$ and $40 \mathrm{~mm}$.

The Aero-triangulation of this project was executed ba using UltraMap AT. Thus an automatically derived high quality tie point collection and computer assisted GCP and checkpoint measurements were introduced into the bundle adjustment. The final adjustment was conducted by BINGO (a softwqare product by GIP, Aalen, Germnany), the embedded least squares engine of our AT solution. 


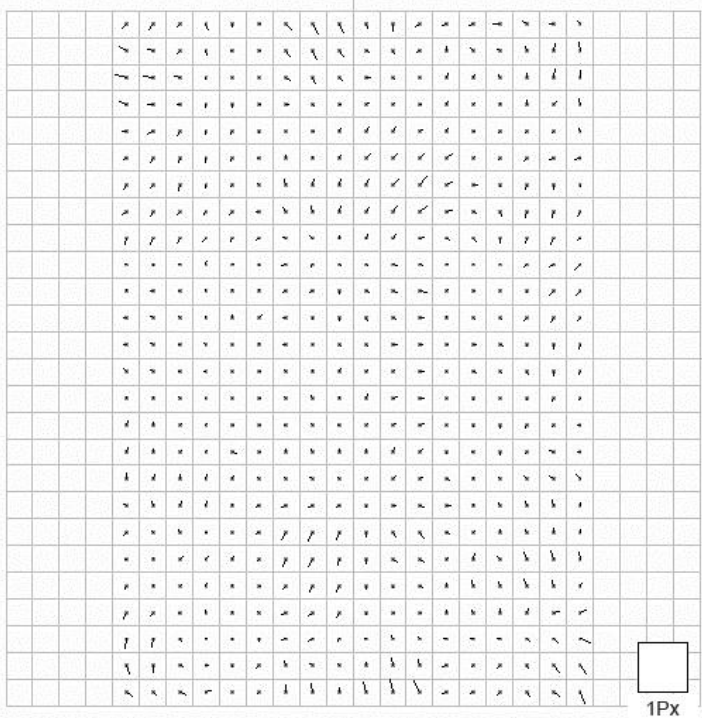

Figure 5: Results from the bundle adjustment: The RMSE image residuals are at $0.8 \mu \mathrm{m}$. The entire number of image positions measured is 58165 from 5525 object points.

\section{Radiometric performance}

The radiometric performance of UltraCam Eagle is particularly supported by the new CCD sensor arrays. This high end component features a dynamic bandwidth of more than $72 \mathrm{~dB}$ and thus an information content of 12 Bit / Pixel. The Pixel size of this sensor is $5,2 \mu \mathrm{m}$ by $5,2 \mu \mathrm{m}$. The new electronic components of the camera are tuned to manage this sensor, the readout the analog signal of the sensor and convert it to digital at a bandwidth of 14 bit. The digital format of the entire processing chain is consequently at the 16 bit level. Figures 6 and 7 illustrates the high dynamic range by an image sample from a high dynamic scene. Bright snow fields and dark shadows show a scene contrast of about 100/1.

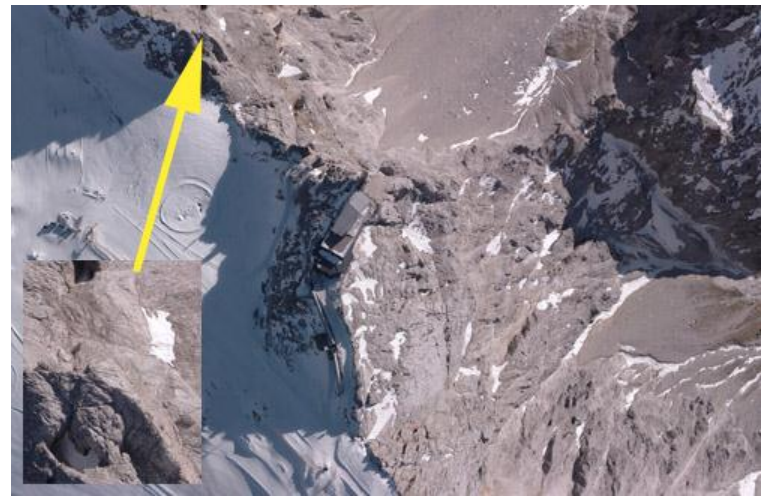

Figure 6: Aerial image taken on Nov-28-2011 above the Dachstein massive in the Austrian Alps. The detail shows bright and dark areas (snow and shadow).
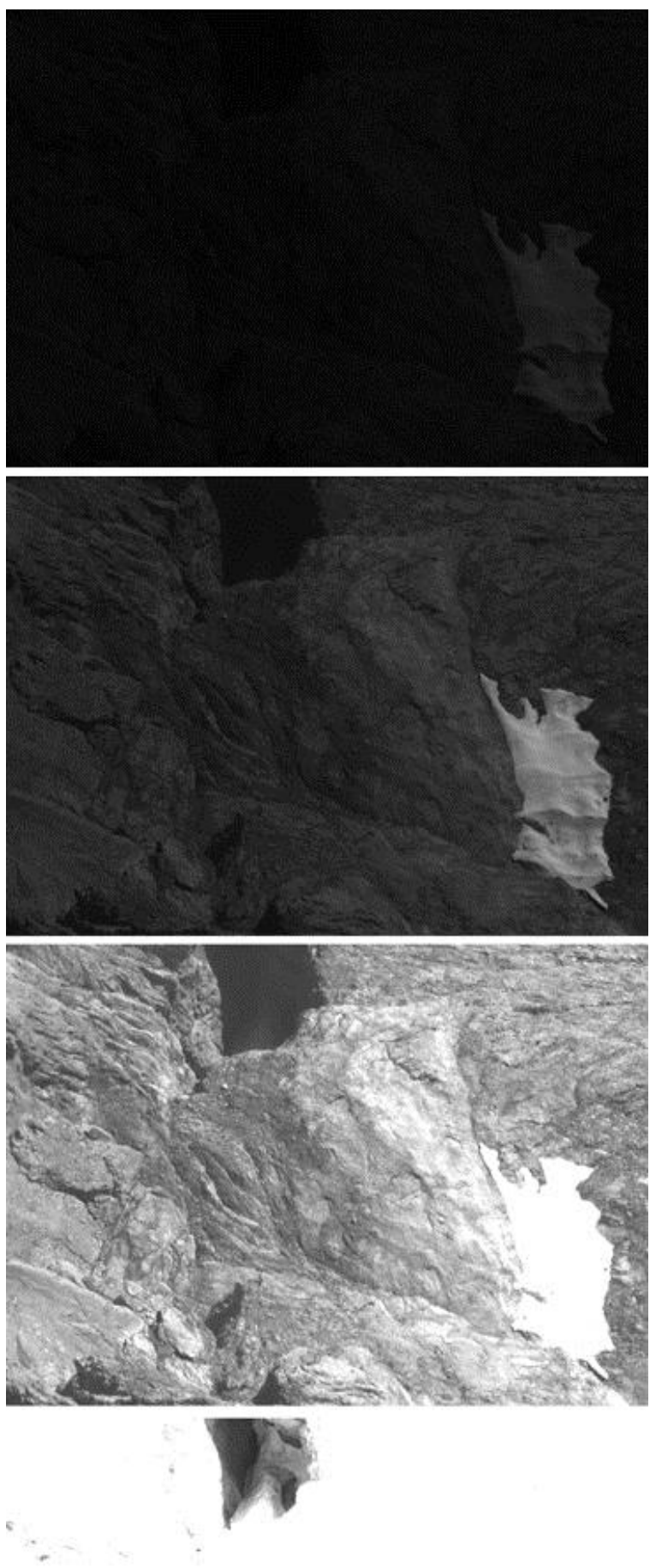

Figure 7: Set of four illustrations of the same detail at different brightness settings (factors 1, 4, 4, and 16 from top to bottom). The difference between dark and bright is about 6,7 bit. 


\section{UltraMap Software}

Sensor management, post-processing and the photogrammetric processing chain are covered by the UltraMap Software product. The integrated workflow offers a state of the art framework which enables the user to exploit a computing environment with a number of CPUs, GPUs and storage components. The development path of the software includes improvements of the post processing (e.g. Monolithic Stitching) and enhanced functionality like aero-triangulation, radiometric adjustment and Dense Matching and Ortho Processing (cf. Fig. 8)..

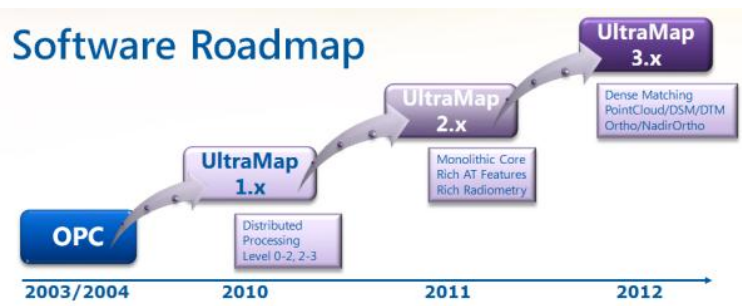

Figure 8: UltraMap Software Roadmap from 2010 to 2012. Image post-processing and photogrammetric processing are part of the integrated workflow.

The new Version 3.0 includes the dense surface based on the Dense Matching technology which was developed as an in house product for Microsoft Bing Maps. Figure 9 and Figure 10 illustrates the results. Aerial images at $7 \mathrm{~cm}$ GSD were used to extract the DSM, process a DSM based ortho image and enables the export of point clouds..

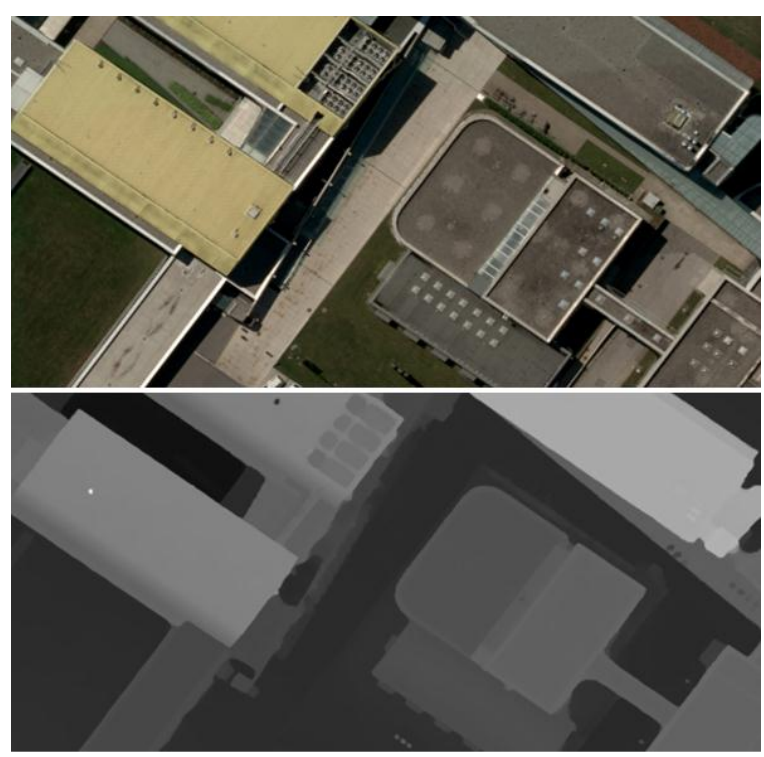

Figure 9: Result from the Dense Matching: DSM based Ortho and DSM (grey level coded) from UltraCam images at $7 \mathrm{~cm}$ GSD.
The roll out of the product during 2012 offers this software technology to the photogrammetric community.

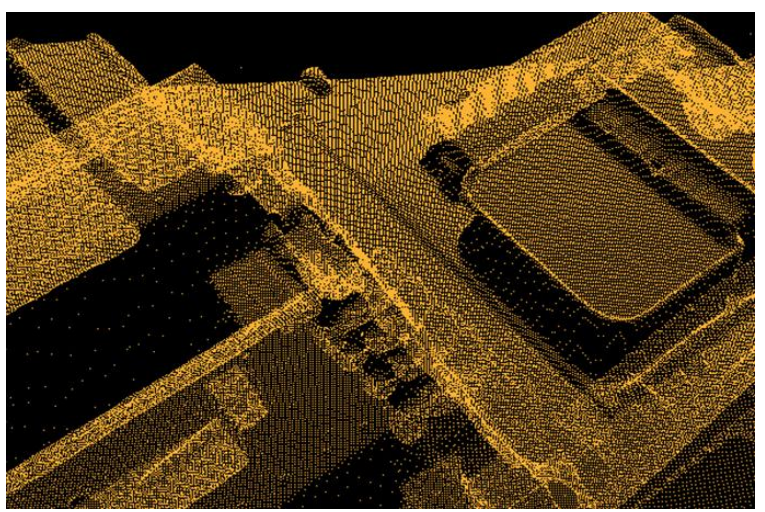

Figure 10: Dense Point Cloud derived from DSM Data (cf. Figure 9)

\section{Conclusions}

The ultra large frame size of the UltraCam Eagle improves the efficiency of the aerial acquisition. This is clearly a benefit to the user and counts during aerial operations.

The radiometric and geometric performance of the camera is at state of the art quality level and enables the user to produce high quality photogrammetry results. Software to operate the sensor and to process the image data is available as well as software for the photogrammetric production. The advantage of this mature technology is well proven and accepted in the community.

\section{References}

Ladstaedter et al, 2010: Monolithic Stitching: One Sensor Geometry For Multiple Sensor Cameras, Proceedings of the American Society for Photogrammetry \& Remote Sensing, 26-30 April, 2010, San Diego, CA

Leberl, F. et al. 2003: The UltraCam Large Format Aerial Digital Camera System, Proceedings of the American Society for Photogrammetry \& Remote Sensing, 5-9 May, 2003, Anchorage, Alaska

Wiechert, A., Gruber M., Ponticelli M. (2011) UltraCam Eagle, the new Super-Large Format Digital Aerial Camera, Proceedings of the American Society for Photogrammetry \& Remote Sensing, 1-5 May, 2011, Milwaukee, WI.

Gruber, M. et al. 2012: UltraCam Eagle, understanding the new Sensor, Proceedings of the American Society for Photogrammetry \& Remote Sensing, 19-23 March 2012, Sacramento, CA. 